\title{
Immunoblot Assay is Still Useful For the Serological Diagnosis of Autoimmune Bullous Dermatosis
}

\author{
D. Bertin ${ }^{1}$, C. Léonnet ${ }^{1}$, P. Berbis ${ }^{2}$, D. Gilbert ${ }^{3}$, M.A. Richard ${ }^{4}$, F. Carsuzaa ${ }^{5}$, J.J. Grob ${ }^{4}$ and \\ S. Desplat-Jégo*, \\ ${ }^{1}$ Laboratoire d'Immunologie, Pôle de Biologie, CHU Conception, Hôpitaux de Marseille, France \\ ${ }^{2}$ Service de Dermatologie, CHU Nord, Hôpitaux de Marseille, France \\ ${ }^{3}$ Laboratoire d'Immunologie, CHU de Rouen, France. \\ ${ }^{4}$ Service de Dermatologie, CHU Timone, Hôpitaux de Marseille, France \\ ${ }^{5}$ Service de Dermatologie, Hôpital d'Instruction des Armées Ste Anne, Toulon, France
}

\begin{abstract}
The detection of autoantibodies to epidermal or basement membrane zone proteins by immunoblot (IB) is useful for the diagnosis and the classification of autoimmune bullous diseases (AIBD). IB using human skin extracts is actually the reference method but A431 cell line is proposed as easier alternate antigen source.

We explored the performances of “A431 IB” in comparison with the reference technique in retrospectively selected patients suffering from well-established bullous pemphigoid $(n=42)$ or pemphigus vulgaris $(n=15)$ and controls $(n=80)$ in order to determine the validity and interest of this simplified IB method.

We demonstrated that in our selected population A431 IB performances are comparable to the reference IB. IB remains semi-quantitative and time-consuming but much more economical and informative than commercially available ELISAs. We support the contention that, in 2012, IB, especially A431 IB, is still useful for the serological diagnosis of AIBD.
\end{abstract}

Keywords: Autoimmune bullous dermatosis, immunoblot, A431 cell line.

\section{INTRODUCTION}

The detection of autoantibodies (auto-Ab) to epidermal or basement membrane zone (BMZ) proteins is useful for the diagnosis and the classification of autoimmune bullous diseases (AIBD). Direct immunofluorescence is used for detecting in vivo bound auto-Ab while indirect immunofluorescence (IIF) allows the detection of circulating auto-Ab in the serum $[1,2]$. However IIF methods are often of limited value in differential diagnosis because they don't allow antigen characterization. In this context, recently commercially available ELISA kits are useful to identify the most frequent reactivities related to BPAg1, BPAg2, desmoglein 1 and desmoglein 3 antigens but they remain expensive tests which target only four antigens. Immunoblotting (IB) improves diagnostic efficiency by enabling recognition of a wide skin antigen panel by using molecular weight separation [3]. Unfortunately, these latter techniques are time-consuming and require different antigen sources to be exhaustive. In order to propose a reliable and labour saving substrate for IB diagnosis of AIBD, Lee has reported in 2000, the human cell line A431, a differentiated adult epidermoid cell line, as

*Address correspondence to this author at the Laboratoire d'Immunologie Pôle de Biologie - Hôpital de la Conception 147, Bd Baille - 13005 Marseille - France; Tel: +33 491383 907; Fax: +33 491383 633;

E-mail: sophie.jego-desplat@ap-hm.fr containing major tissue antigens of AIBD [4]. However, Lee et al. tested a very few patients and did not compare the method with IIF, reference IB with human skin extracts or ELISA. In our study, we explored the performances of "A431 IB" in comparison with the reference techniques in patients suffering from well established bullous pemphigoid (BP) or pemphigus vulgaris (PV) in order to determine the validity and interest of this simplified IB method.

\section{REPORT}

We have retrospectively included 42 patients with BP (22 males and 20 females with mean age of 79.9 years) and 15 with PV (6 males and 9 females with mean age of 57.6 years). As controls, 21 patients with non auto-immune dermatosis (eczema, prurigo, and second or third-degree burn) (10 males and 11 females with mean age of 64.8 years) and 59 healthy blood donors (25 males and 34 females with mean age of 42.6 years) were enrolled. All patients have given their informed consent and have been examined in three departments of dermatology from the AIBD centre of competence of the south of France. Clinical data associated with histological skin examination coupled with direct immunofluorescence and IIF have permitted to establish a certainty diagnosis of BP or PV. IIF and A431 IB were all performed in the immunology laboratory of the CHU of Marseilles. IIF was performed with a standard technique by using monkey oesophagus as substrate and a monkey-adsorbed 
conjugate [5]. A431 IB was conducted according to the work by Lee (Lee, 2000). Reference IB was realized in the AIBD French reference laboratory of the Rouen University Hospital as previously described [6]. For this study, both IB tests were considered as positive when antibodies against: 1 - the 230 (BPAg1) and/or 180 (BPAg2) kDa antigens for the group of BP or 2- the 130 (desmoglein-3) and 160 (desmoglein-1) kDa antigens for the group of PV (see Fig. (1) for illustration), were detected. In case of discrepancies between the two IB, we completed the serum analysis by commercially available BP-230, BP180-NC16A, desmoglein-1 or desmoglein-3 ELISA (MBL, Nagoya, Japan).

Results of IIF and IB are summarized in Table 1. We found a positive test by IIF in $75.4 \%$ of AIBD patients.

Among BP patients, 32 were positive for reference IB and 34 for A431 IB. In both tests, the most frequent detected reactivity was against the $180 \mathrm{kDa}$ antigen alone (respec- tively $15 / 32$ and 18/34). It is worthy of note that IB was positive for $7 \mathrm{BP}$ patients with negative IIF: 3 were positive for both IB tests, 2 were positive only for reference IB test and 2 others were positive only for A431 IB.

Among PV patients, 7 were positive for reference IB and 6 for A431 IB. In both tests, the most frequent detected reactivity was against the $130 \mathrm{kDa}$ antigen alone (respectively 6/15 and 4/15). Interestingly, IB was positive for $1 \mathrm{PV}$ patient despite IIF was negative: this positivity was observed with A431 IB only.

In control groups all IB tests were negative even a burnt patient exhibiting intercellular staining in IIF. The sensitivity of A431 IB and reference IB were $80.9 \%$ and $76.2 \%$ for the diagnosis of BP and $40.0 \%$ and $46.6 \%$ for the diagnosis of $\mathrm{PV}$, respectively. The specificity of the two tests was equal for both groups of diseases and reached $100 \%$ since all IB were negative in the control groups. Total discrepant results

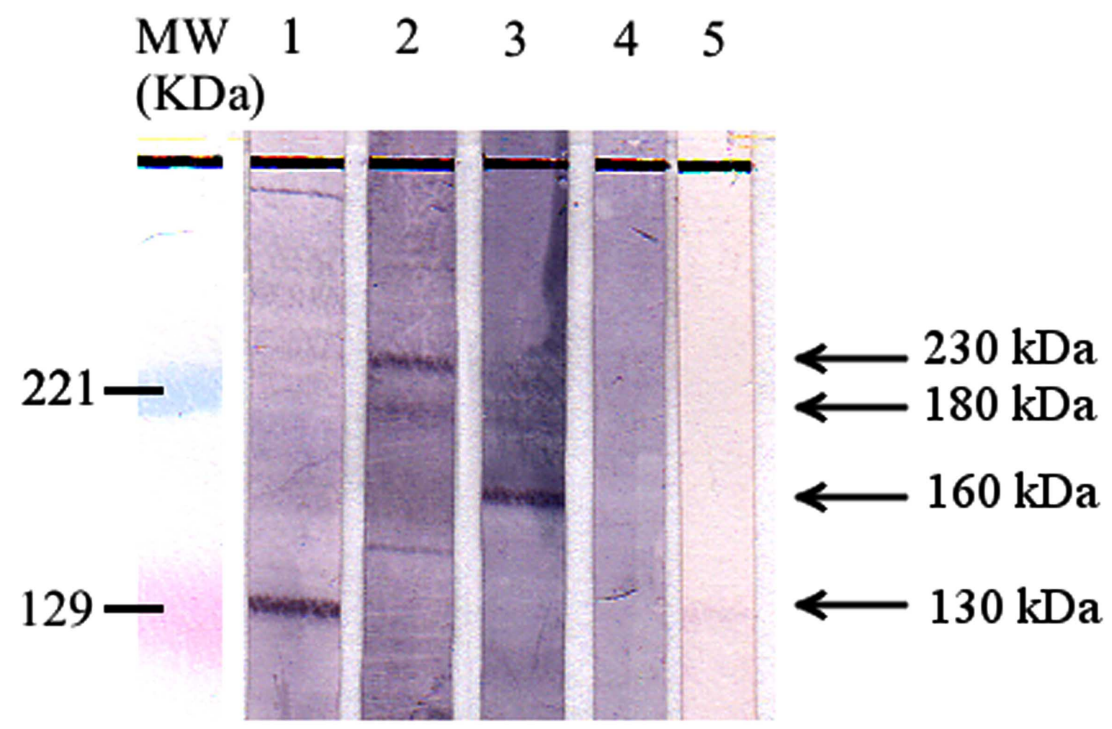

Fig. (1). Immunoblot detection of bullous pemphigoid and pemphigus antigens with A431 cell extract.

Lanes 1 to 3: sera reactivities against $130 \mathrm{kDa}$ antigen (1), 180 and $230 \mathrm{kDa}$ antigens (2) and $160 \mathrm{kDa}$ antigen (3) respectively - Lane 4: Negative control serum - Lane 5: PBS negative control

Table 1. Comparison of Indirect Immunofluorescence and Immunoblot in Serological Diagnosis of Auto-immune Bullous Dermatosis.

\begin{tabular}{|c|c|c|c|c|}
\hline $\begin{array}{c}\text { Nosological group } \\
\text { (According to Clinical and Histological/DIF Data) }\end{array}$ & $\begin{array}{c}\text { Positive IIF } \\
(\%)\end{array}$ & \multicolumn{2}{|c|}{$\begin{array}{c}\text { Positive Human Skin Extract IB } \\
\text { Assay }\end{array}$} & $\begin{array}{c}\text { Positive A431 Cell Extract IB } \\
\text { Assay }\end{array}$ \\
\hline \hline $\begin{array}{c}\text { Bullous pemphigoid } \\
(\mathrm{n}=42)\end{array}$ & $\begin{array}{c}32 \\
(76.2)\end{array}$ & 27 & 5 & Positive IIF \\
\hline $\begin{array}{c}\text { Pemphigus vulgaris } \\
(\mathrm{n}=15)\end{array}$ & $\begin{array}{c}11 \\
(73.3)\end{array}$ & 7 & 0 & 5 \\
\hline $\begin{array}{c}1 \\
(\mathrm{n}=21)\end{array}$ & $\begin{array}{c}\text { Negative IIF } \\
(4.8)\end{array}$ & 0 & 0 \\
\hline $\begin{array}{c}0 \\
\text { Non auto-immune dermatosis } \\
(\mathrm{n}=59)\end{array}$ & 0 & 0 & 0 \\
\hline
\end{tabular}

DIF: Direct ImmunoFluorescence - IB: Immunoblot - IIF: Indirect Immunofluorescence 
between the two IBs concerned $8.4 \%$ of sera (9/137). The kappa coefficient was 0.81 showing a good concordance between the two tests. It is noteworthy that ELISA tests performed in case of IB discrepancies supported the established diagnosis in all cases. In fact in the BP group, all IB discrepant serum were positive for anti-BPAg1 and/or antiBPAg2 while in the PV group, all serum were positive for anti-Dsg1 and/or anti-Dsg3.

\section{DISCUSSION}

We report here the performances of IB using A431 cell extracts in comparison with the reference IB which requires human epidermis and dermis extracts. The present findings confirm that A431 cell extracts contain epidermal and dermo-epidermal junction components necessary to detect major auto-antibodies involved in AIBD. We show that the two tests have $100 \%$ specificity and a comparable sensitivity close to $80 \%$ for BP with a good concordance. However concordance is not total, showing that each IB can diagnose a few cases that the other cannot. We suggest that the few discrepancies between the two IB tests were mostly due to some differences in antigen source but also in reagents (for example, secondary detecting antibody were not the same) and procedure.

Testing sera with IB has permitted to detect reliable autoantibodies in 7 patients with BP and 1 patient with PV displaying negative IIF. IB has also allowed excluding an IIF false positive due to extensive burn. We have to keep in mind that we have considered only BP230, BP180, desmoglein-1 or desmoglein-3 antigen detection. Then the sensitivity of the tests could be underestimated since AIBD may involve various other auto-antigens [7]. However this point underlines the interest of IB testing since it is able to detect other specificities that ELISA doesn't. For example, we have to mention the ability in our hands of A431 IB test to detect relativities against periplakin and envoplakin in patients suffering from paraneoplastic pemphigus (data not shown). The poor sensitivity of A431 extracts for PV diagnosis has been reported with other antigen sources [8] since the majority of desmoglein auto-immune epitopes appear to be conformational and therefore destroyed by SDS molecules. In our study, specificity of IB is excellent. We are not in accordance with recently published results [9] showing significant numbers of normal healthy subjects (59\%) with circulating auto-antibodies to BMZ proteins by immunoblot but very few studies have assessed BMZ antibodies in normal subjects. However a simple explanation could be the difference of serum dilution used in the two works: we used 1:40 dilution for our IB tests while Nemesha Desai's team used 1:2, 1:5 and 1:10 dilutions. The main advantage of A431 cell line relies on its simplicity to obtain and to manage. The use of this easy-to-grow in vitro cell line exempts the biologist from saving human skin samples issued from plastic surgery or human amniotic membrane from obstetric department [10]. Moreover, in our hands, this method allows a good reproductibility as shown by positive and negative internal quality controls used in each experiment.

IB method even with A431 cell extracts remains semiquantitative and time-consuming but much more economical and more informative than commercially available ELISAs which are not yet able to test a large panel of recombinant skin antigens. In conclusion we support the contention that in 2012 immunoblot assay, especially A431 IB, is still useful for the serological diagnosis of AIBD.

\section{CONFLICT OF INTEREST}

The authors confirm that this article content has no conflicts of interest.

\section{ACKNOWLEDGEMENTS}

\author{
None declared
}

\section{REFERENCES}

[1] Chan Y-C, Sun Y-J, Ng PP-L, et al. Comparison of immunofluorescence microscopy, immunoblotting and enzyme-linked immunosorbent assay methods in the laboratory diagnosis of bullous pemphigoid. Clin Exp Dermatol 2003; 28(6): 651-6.

[2] Harman KE. New laboratory techniques for the assessment of acquired immunobullous disorders. Clin Exp Dermatol 2002; 27(1): 40-6.

[3] Ghohestani R, Kanitakis J, Nicolas JF, et al. Comparative sensitivity of indirect immunofluorescence to immunoblot assay for the detection of circulating antibodies to bullous pemphigoid antigens 1 and 2. Br J Dermatol 1996;135(1): 74-9.

[4] Lee CW. An extract of cultured A431 cells contains major tissue antigens of autoimmune bullous diseases. Br J Dermatol 2000; 143(4): 821-3.

[5] Jiao D, Bystryn JC. Sensitivity of indirect immunofluorescence, substrate specificity, and immunoblotting in the diagnosis of pemphigus. J Am Acad Dermatol 1997; 37(2 Pt 1): 211-6.

[6] Joly P, Gilbert D, Thomine E, et al. Relationship between the in vivo localization and the immunoblotting pattern of anti-basement membrane zone antibodies in patients with bullous pemphigoid. Arch Dermatol 1997; 133(6): 719-24.

[7] Mihai S, Sitaru C. Immunopathology and molecular diagnosis of autoimmune bullous diseases. J Cell Mol Med 2007; 11(3): 46-81.

[8] Pas HH. Immunoblot assay in differential diagnosis of autoimmune blistering skin diseases. Clin Dermatol 2001; 19(5): 622-30.

[9] Desai N, Allen J, Ali I, et al. Autoantibodies to basement membrane proteins BP180 and BP230 are commonly detected in normal subjects by immunoblotting. Australas. J Dermatol 2008; 49(3): $137-41$.

[10] Grootenboer-Mignot S, Descamps V, Picard-Dahan C, et al. Place of human amniotic membrane immunoblotting in the diagnosis of autoimmune bullous dermatoses. Br J Dermatol 2010; 162(4): 74350 . 